\section{Sleep Quality in Semi-Professional Male Basketball Players in Bermuda}

S Roopchand-Martin ${ }^{1}$, R Bennett ${ }^{2}$, A Facey ${ }^{1}$, S CrearyYan $^{1}$, A Mansingh ${ }^{1}$

${ }^{1}$ Mona Academy of Sport, Faculty of Sport, The University of the West Indies, Kingston 7, Jamaica.

${ }^{2}$ St. Ann's Bay Hospital, Seville Road, St. Ann's Bay, Jamaica.

\section{Corresponding Author}

Dr. Sharmella Roopchand-Martin

Mona Academy of Sport

Faculty of Sport

The University of the West Indies,

Kingston 7, Jamaica.

Email address:

sharmella.roopchandmartin@uwimona.edu.jm

\section{DOI: $\underline{10.48107 / C M J 2021.04 .011}$}

Copyright: This is an open-access article under the terms of the Creative Commons Attribution License which permits use, distribution, and reproduction in any medium, provided the original work is properly cited.

(C)2021 The Authors. Caribbean Medical Journal published by Trinidad \& Tobago Medical Association.

\section{ABSTRACT}

Objectives: This study sought to determine the quality of sleep using the Pittsburgh Sleep Quality Index (PSQI), the presence of sleepiness using the Epworth Sleepiness Scale (ESS) and the association between sleep quality and sleepiness in basketball players in Bermuda.

Methods: Once ethical approval was granted, players were recruited from the Bermuda Basketball Association League. All participants completed the PSQI and the ESS questionnaires based on their recollection of events as they occurred over the previous 30 days. Their responses were analysed using the IBM SPSS version 19 for Windows.

Results: A total of 71 subjects, mean age $24.96 \pm 3.19$ years, participated in this study. The mean PSQI score was $7.8 \pm 4.7$ (scores of 5 or more indicate poor sleep quality). Thirty percent of players rated their sleep quality as fairly bad to very bad. The mean sleepiness score was $7.35 \pm 4.17$ and over $60 \%$ of persons surveyed had more than normal levels of sleepiness. There was a significant correlation between sleep quality and sleepiness; 0.61 ( $p$ $<0.01$ ), as well as a correlation between age and Global PSQI which had a score of $0.31(p<0.01)$.

Conclusion: Basketball players in Bermuda are experiencing less than optimal sleep. Insomnia was among the most popular self-reported cause of sleep disturbances. Further research is required in this population, exploring causal factors for poor sleep quality.

Key words: Athletes, Pittsburgh Sleep Quality Index, Epworth Sleepiness Scale, Sleep Quality.

\section{INTRODUCTION}

Optimal athletic performance is the primary goal that drives the sporting fraternity. The fundamental principle of the "training response" focuses on a balance between stress, fatigue and recovery ${ }^{1}$, which must be systematically followed. While significant research has been aimed at perfecting the first two components across the spectrum of sports, sleep has been less investigated. Sleep has been shown to be arguably one of the best forms of recovery available to athletes and plays an 
important role in the adaptation of training stimulus; with resultant improvements in skill acquisition, physical performance and mood variables ${ }^{2-4}$. In addition to growing evidence suggesting that athletes do not obtain sufficient sleep, assessments of sleep quality in competitive athletes reveals a substantial prevalence of poor sleep quality ${ }^{4-11}$. A review of sleep quality in elite athletes has reported a high overall prevalence of insomnia symptoms as reflected by longer sleep latencies, greater sleep fragmentation, nonrestorative sleep, and excessive daytime fatigue ${ }^{11}$. Additionally, mean Pittsburgh Sleep Quality Index scores between 5 and 6 (scores $>5$ indicate poor sleep quality) were reported in five studies conducted in athletes involved in team and individual sports ${ }^{11}$. It has been reported that athletes may require more sleep than non-active individuals to allow for adequate recovery and adaptation between bouts of exercise, roughly 9 to 10 hours per day ${ }^{12}$. Despite this, there are currently no specific guidelines regarding sleep duration or quality for athletes.

Athletes encounter a wide array of factors that may serve as barriers to good sleep quality and quantity. These include lengthy travel to competitions, jet lag, training schedules both in terms of time of day and duration, precompetition anxiety and the infamous record of injuries sustained $^{4-5,11,13}$. A negative or decreased response in motor and cognitive performance, emotional stability and a range of metabolic and immunologic processes are often observed in athletes who are sleep-deprived ${ }^{2,14}$. Poor sleep quality, particularly during high training loads and competition period, has been identified as a marker of under-recovery and an early sign of overreaching, resulting in its recommended monitoring through the progressive stages of the stress/fatigue state continuum 15-16. The aforementioned signifies the importance of sleep in the recovery process and provides a potential link between sleep and the direct and indirect effects on athletic performance.

Whilst a general trend of poor sleep quality has been reported for elite athletes it is also important to note that there is inter-sport variability ${ }^{11}$. Sports where early training occurred, those where aesthetics was an important factor and those involving female athletes often reported higher levels of insomnia ${ }^{11}$. Additionally, studies have shown significant within subject variability in sleep quality which may be related to the individual differences in coping with sport specific factors that can affect sleep like pre-competitive stress and circadian disruptions ${ }^{11}$. These differences speak to the need to conduct sport specific studies.

Basketball is a highly competitive sport which requires a high level of cardiovascular fitness, agility, speed, power and vigilance. Athletes rely heavily on glycogen reserves and it is during sleep that glucose is metabolised and stored in muscles in the form of glycogen. Sleep deprivation slows this process by as much as $30-40 \%{ }^{17}$. Peak oxygen consumption was shown to be reduced after partial sleep deprivation compared with normal sleep conditions ${ }^{18}$ and decreased accuracy in serving was shown in sleep deprived tennis players ${ }^{19}$. A pilot study in soccer players showed no difference in sleep quality and quantity on days when the players engaged in early evening, high intensity training sessions versus days where there was no evening training ${ }^{20}$. Research exploring non-invasive methods to improve sleep quality have shown that sleep extension (at least 10 hours in bed at night) in basketball players showed improvements in athlete's sprint time, reaction time and three-point field goals ${ }^{4}$. The effect of red-light exposure on sleep quality and endurance performance in Chinese female basketball players showed a correlation between changes in global PSQI and serum melatonin levels $(r=-0.695, P=.006)^{21}$.

The Bermuda National Basketball team is chosen from the pool of players that participate in the Bermuda Basketball Association League. Over the years, the national team has not had much success internationally. Given the literature that indicates that sleep is one factor that could impact on sport performance this study sought to explore self-reported sleep quality in semi-professional basketball players in Bermuda and to determine whether there was a relationship between sleep quality and daytime sleepiness in this group.

\section{METHODS}

On obtaining ethical approval to conduct this study from the Ethics Committee of the University of the West Indies, Mona, Jamaica, all male basketball players, from ages 18 to 30 years, who participated in the Bermuda Basketball Association League and who provided written consent to participate were included. Participants were excluded if they reported musculoskeletal injuries, pain or 
any medical conditions that required medications which may affect sleep. A total of 71 players, from a targeted pool of 80 , participated. This slightly exceeded the targeted sample size of 67 which was based on a $5 \%$ margin of error, $95 \%$ confidence interval and $50 \%$ response rate. All participants completed the Pittsburgh Sleep Quality Index (PSQI) and Epworth Sleepiness Scale (ESS).

\section{Outcome Measures}

The Epworth Sleepiness Scale is a standardized, selfreport questionnaire that assesses daytime sleepiness. The instrument required the participant to rate the likelihood of falling asleep, on a scale of increasing probability from $0-3$, in eight different situations that most people engage in during their daily activities. The scale does not specify a time, instead it requires that persons give an average rating of how sleepy they feel in daily life. The total ESS score is the sum of 8 item-scores and can range between 0 and 24 with higher scores indicating a higher level of daytime sleepiness. Scores in the range of 0-9 reflects normal levels of sleepiness ${ }^{22}$.

The Pittsburgh Sleep Quality Index is a standardized questionnaire used to measure the quality and patterns of sleep. It is a self-report questionnaire that assesses sleep quality over a one-month interval. It consists of nineteen individual items, creating seven components that produce a global score. The component scores consist of subjective sleep quality, sleep latency, sleep duration, habitual sleep efficiency, sleep disturbances, use of sleep medication and daytime dysfunction. Each item was weighted on a 0-3 interval scale, which provided a score ranging from 0-21, where lower scores denoted a healthier sleep quality. Scores of 5 and above are indicative of poor sleep quality ${ }^{23}$. Both tools have been found to have good internal consistency and reliability in the adult population ${ }^{22-23}$.

\section{Data Analysis}

Statistical analysis was executed using IBM SPSS statistical software version 19 for Windows. Descriptive statistics were performed to analyse sleep quality and sleepiness scores on the Pittsburgh Sleep Quality Index and the Epworth Sleepiness Scale. The raw data was normally distributed and therefore a Pearson's Correlation Coefficient was used (alpha level 0.05) to investigate the relationships between age and global PSQI score and global PSQI and ESS scores. An analysis of variance was done to evaluate whether there was a difference in sleepiness scores and medication use.

\section{RESULTS}

All basketball players who met the criteria for participation (71) completed both questionnaires. Ages ranged from 18-30 years, with a mean (SD) of $24.96 \pm$ 3.19. Most of the participants (73.2 \%) were found to have levels of daytime sleepiness that exceeded normal levels, with $33.8 \%$ of that cohort reporting "Higher Normal" daytime sleepiness. Only $1.4 \%$ was classified as having severe excessive daytime sleepiness (Figure 1). The individual responses to items on the ESS showed higher sleepiness scores for sitting and reading a book $(1.4 \pm 1.1)$, watching television $(1.5 \pm 1)$ and lying down to rest in the afternoon $(1.9 \pm 0.9)$. All other items had a mean score of less than one (Table 1 ).

\section{Table 1: The mean and standard deviation for each item on the Epworth Sleepiness Scale $(\mathbf{N}=71)$}

\begin{tabular}{|l|c|c|}
\hline Items on the Epworth Sleepiness Scale & Mean & S.D. \\
\hline $\begin{array}{l}\text { How likely are you to doze off or fall asleep in the following situa- } \\
\text { tions, in contrast to feeling just tired? }\end{array}$ & 1.4 & 1.1 \\
\hline Sitting and Reading & 1.5 & 1.0 \\
\hline Watching TV & 0.7 & 0.9 \\
\hline Sitting inactive in public space & 0.8 & 0.8 \\
\hline Passenger in a car for an hour without break & 1.9 & 0.9 \\
\hline Lying down to rest in the afternoon if circumstances permit & 0.3 & 0.6 \\
\hline Sitting and talking to someone & 0.6 & 0.7 \\
\hline Sitting quietly after lunch with no alcohol intake & 0.1 & 0.4 \\
\hline In a car while stopped for a few minutes in traffic & & \\
\hline
\end{tabular}


Figure 1: The percentage of participants in each category of sleepiness based on their total score on the Epworth Sleepiness Scale ( $\mathbf{N}=71)$

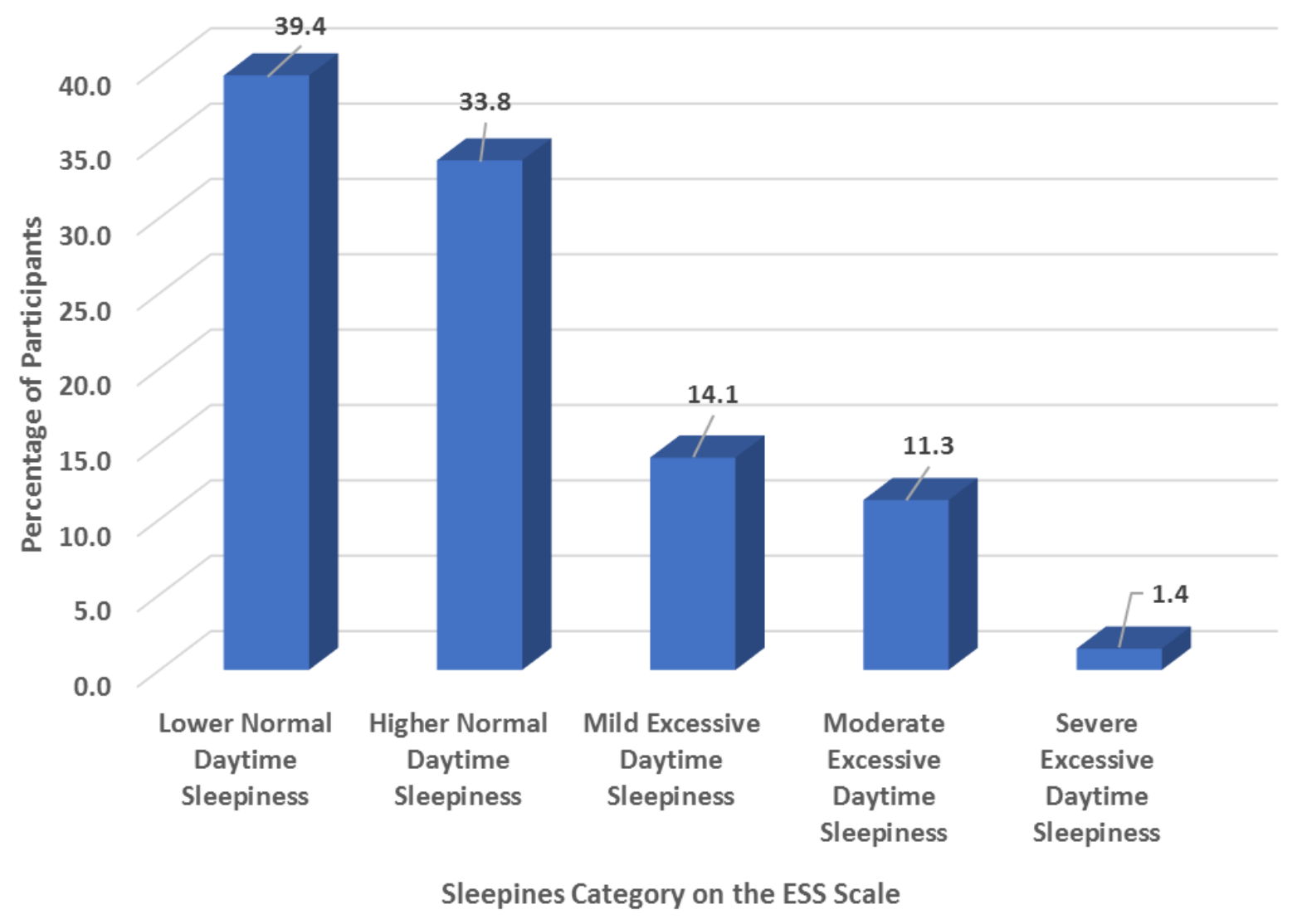

The mean global score of the PSQI was 7.8 \pm 4.7. Approximately $75 \%$ of the players did not state any complaints of sleeping difficulties, while less than $1 \%$ reported illnesses. A high number of subjects on the PSQI reported waking in the middle of the night/early morning and waking to use the bathroom three (3) or more times per week and $71.8 \%$ (51) responded to taking medication at some point in the past month to help them sleep. Twenty-eight (39.4\%) of the participants took sleep medication on a weekly basis (Table 2). Forty five percent of participants reported having good sleep for most of the days weekly, while $7 \%$ reported having poor sleep more than 3 days per week (Table 2). A total of $40.8 \%$ (29) of participants reported sleeping seven or more hours per night whilst $59.2 \%$ (42) got less than six hours of sleep each night.

The correlation between Global PSQI and the ESS was found to be statistically significant at $0.61(p<0.01)$, as well as the correlation between age and Global PSQI which had a correlation score of $0.31(p<0.01)$. Partici- pants who took medications to sleep either once or twice per week or three or more times per week had significantly higher daytime sleepiness scores (11.00 \pm 3.44 and $10.50 \pm 2.59$ ) compared to those who took medications less than once per week or not at all $(7.70 \pm 3.34$ and $3.54 \pm 3.16)(p<0.05)$.

\section{DISCUSSION}

This study aimed to identify the quality of sleep and level of daytime sleepiness present in basketball players in Bermuda using the Pittsburgh Sleep Quality Index (PSQI) and the Epworth Sleepiness Scale (ESS). The mean PSQI score on this study was above five $(7.8 \pm 4.7)$ thus indicating poor sleep quality. In fact, at least $30 \%$ of players rated their sleep quality as fairly bad to very bad. Sleep quality was significantly correlated with daytime sleepiness and older persons had more problems with sleep. This is a cause for concern since it is quite likely that the athletes in this study may be experiencing some of the adverse effects from poor sleep quality reported in the literature such as decreased response in motor and 
Table 2: Participants responses to each item on the PSQI $(\mathbf{N}=71)$

\begin{tabular}{|l|c|c|c|c|}
\hline Items on the Pittsburg Sleep Quality Index & $\begin{array}{l}\text { Not during } \\
\text { the past } \\
\text { month }\end{array}$ & $\begin{array}{l}\text { Less than } \\
\text { once a } \\
\text { week }\end{array}$ & $\begin{array}{l}\text { once or twice } \\
\text { per week }\end{array}$ & $\begin{array}{l}\text { Three or } \\
\text { more times } \\
\text { per week }\end{array}$ \\
\hline $\begin{array}{l}\text { During the past month, how often have you had } \\
\text { trouble sleeping because you: }\end{array}$ & \multicolumn{2}{|l|}{ Number of Respondents } \\
\hline Cannot get to sleep within 30 minutes & 33 & 7 & 11 & 20 \\
\hline Wake up in the middle of the night or early morning & 15 & 8 & 13 & 35 \\
\hline Have to get up to use the bathroom & 17 & 7 & 14 & 33 \\
\hline Cannot breathe comfortably & 54 & 8 & 4 & 5 \\
\hline Cough or snore loudly & 37 & 6 & 11 & 17 \\
\hline Feel too cold & 44 & 16 & 6 & 5 \\
\hline Feel too hot & 38 & 15 & 11 & 7 \\
\hline Have bad dreams & 44 & 17 & 9 & 1 \\
\hline Have pain & 38 & 22 & 8 & 3 \\
\hline $\begin{array}{l}\text { During the past month, how often have you taken } \\
\text { medicine (prescribed or "over the counter") to help }\end{array}$ & 20 & 33 & 12 & 6 \\
\hline $\begin{array}{l}\text { During the past month, how often have you had } \\
\text { trouble staying awake while driving, eating meals, or } \\
\text { engaging in social activity }\end{array}$ & 39 & 16 & 11 & 5 \\
\hline $\begin{array}{l}\text { During the past month, how much of a problem has it } \\
\text { been for you to keep up enthusiasm to get things }\end{array}$ & 42 & 16 & 8 & 5 \\
\hline $\begin{array}{l}\text { During the past month, how would you rate your sleep } \\
\text { quality overall? }\end{array}$ & 32 & 18 & 16 & 5 \\
\hline
\end{tabular}

cognitive performance and emotional stability ${ }^{2,14}$. The results of this study are like that reported in the literature where athletes were shown to have poor sleep quality ${ }^{4-11}$. Six studies that also used the PSQI for individual and team sports also reported mean scores of 5 and above ${ }^{11}$.

Over $60 \%$ of persons surveyed had higher than normal levels of sleepiness. It was noted that activities that require increased mental or cognitive activity like reading a book were found to be those more likely to evoke daytime sleepiness. It is possible that if this is not addressed increased levels of daytime sleepiness may not only affect athletic performance but also performance in their regular jobs and overall quality of life. Older persons were found to have poorer sleep quality, and this may have been related to other stressors such as work, families to take care off and bills to pay. None of the participants in this study were employed to play basketball only and therefore held regular jobs in addition to playing the sport. The demands of managing multiple activities may have led to reduced time for sleep which would be different to what pertains for elite athletes whose full-time job is playing their sport. In fact, more than $50 \%$ of the athletes in this study were getting less than 6 hours sleep at nights which was significantly less than the recommended nine to ten hours to allow for adequate recovery and adaptation between bouts of exercise $^{12}$. This is a concern because inadequate recovery can lead to increased injury risk. The multiple factors that impact on sleep quality were not considered for this study since the focus was only on documenting sleep quality and not on examining causal relationships. It is clear however from the findings that further investigations into these factors is required for this population.

It is important to note that like the Bermudan basketball players many national athletes for both team and individual sports in the Caribbean region work regular jobs and engage in sports training after work, so sleep deficits may also be a serious concern for these individuals. The literature has indicated that elite athletes suffer from poor sleep quality ${ }^{11}$ but one must wonder if 
this may be even more so for some of the elite level athletes in our region. Sleep and sport performance is a relatively new area of focus and given the paucity of data on sleep among Caribbean athletes it is important for future studies in the region to investigate some of these relationships. Researchers need to examine associations between sleep quality, sport performance, injury occurrence and recovery in athletes in different sports in the region. Studies must also be designed to identify factors that may be leading to poor sleep quality in athletes so that appropriate interventions can be developed.

Fifty-one respondents $(71.8 \%)$ were noted to have taken medication to aid sleep in the past month. The frequency of taking medications ranged from less than once per week to up to three times per week. The persons who took sleep medications on a weekly basis also had higher daytime sleepiness levels than those who took medications less than once per week. This finding is important since many subjects reported having some amount of difficulty undertaking their daily activities. This was probably related to a combination of the medication together with poor sleep quality and inadequate hours of sleep. These persons may be placing themselves at risk for serious injury both at work (depending on the nature of their job) and during training and competition due to reduced levels of alertness. Furthermore, for athletes, use of some medications can result in positive drug tests and penalties. Further studies should be conducted to explore the types of medications being used by these athletes and whether they are prescribed or unprescribed.

Insomnia (unable to fall asleep within 30 minutes and waking in the middle of the night or early morning), waking to use the bathroom and coughing or snoring loudly were the main reasons for sleep disturbance in the respondents who fell in the severely disrupted/ sleep quality group. These results are in line with previous studies and therefore augment the fact that sleep in many athletes may be suboptimal ${ }^{7,8}$.

Contrary to public opinion, athletes experience disrupted or poor-quality sleep at higher rates than non-athletes and may have higher rates of sleep disorders ${ }^{2}$. Though this study only examined sleep in the past month, most of the players reported that their sleep pattern had been the same for years. Sleep hygiene was not explored in this study however, it is possible that many of the sleep disturbances being experienced may be related to poor sleep hygiene. The coughing and loud snoring could be indicative of other pathology, including sleep apnoea that may require further investigation. Insomnia may be related to mental health disorders, such as depression and anxiety. The findings of this study indicate that sports medicine practitioners should include proper screening for sleep quality in the pre-participation medical evaluation of athletes. For some this may require conducting sleep studies to investigate possible sleep apnoea, but at minimum sleep quality and sleep hygiene should be examined and appropriate recommendations/ referrals made. The small sample size for this study limits the generalizability of the findings and the results must be interpreted with caution however it highlights the definite need for more research in this area.

\section{CONCLUSION}

This study has shown that basketball players in Bermuda are experiencing less than optimal sleep. This study did not evaluate causal factors for sleep problems however, based on the percentage that has greater than normal levels of daytime sleepiness and are using medications to help them sleep there is a definite need for further exploration of causal factors. The study also points to a need for Sports Medicine practitioners to include screening for sleep quality and sleep hygiene in the evaluation of athletes and to collaborate with the sporting bodies to deliver educational sessions to the athletes regarding sleep.

Ethical Approval Statement: Ethical approval to conduct this study was obtained from the UWI Mona Campus Ethics Committee

Conflict of Interest Statement: The authors have no competing interest

Informed Consent Statement: All participants provided consent to participate in this study

Funding Statement: This research was self-funded Author Contributions: Dr. Sharmella Roopchand Martin and Dr Ricard Bennett were involved in the Conception/ Design of the project, Analysis, Interpretation, and critical revision of the paper. Dr. Bennett was also involved in data collection. Alison Facey and Shara Creary-Yan were 
involved in analysis, interpretation, and critical revision of the paper and Dr. Akshai Mansingh was involved in Conception/Design of the project and interpretation and critical revision of the paper

\section{REFERENCES}

1. Pearce PZ. A practical approach to the overtraining syndrome. Curr Sports Med Rep 2002; 1: 179-183.

2. Samuels $C$. Sleep, recovery, and performance: The new frontier in high performance athletics. Neurol. Clin. 2008; 26: 169-180.

3. Venter RE. Role of sleep in performance and recovery of athletes: A review article. S. Afr. J. Res. Sport Phys. Educ. Recreation 2012; 34: 167-184.

4. Mah CD, Mah KE, Kezirian E J, Dement WC. The effect of sleep extension on the athletic performance of collegiate basketball players. Sleep 2011; 34 (7): 943-950.

5. Sargent C, Halson SL, Roach GD. Sleep or swim? Early morning training severely restricts the amount of sleep obtained by elite swimmers. Eur J Sport Sci 2014; 14: 831-15.

6. Birge ME. Sleep quality and quantity of Portland State University intercollegiate student athletes. ProQuestDissertation 2014. Portland State University.

7. Knufinke $M$, Nieuwenhuys $A$, Geurts $S A E$, Coenen $A$ $M L$, Kompier MAJ. Self- Reported Sleep Quantity, Quality and Sleep Hygiene in Elite Athletes. J Sleep Res 2018; 27: 78-85.

8. Leeder J, Glaister M, Pizzaferro K, Dawson J, Pedlar C. Sleep duration and quality in elite athletes measured using wrist actigraphy. Journal of Sport Sci 2012; 30 (6): 514-545.

9. Swinbourne R, Gill N, Vaile J, Smart D. Prevalence of poor sleep quality, sleepiness and obstructive sleep apnoea risk factor in athletes. Eur J Sport Sci 2016; 16 (7) 850-858.

10. Tsunoda K, Hotta K, Mutsuzaki H, Tachibana K, Shimizu, $Y$ et al. Sleep status in male wheelchair basketball players on a Japanese national team. $J$ Sleep Disord Ther 2015; 4: 210-214.

11. Gupta L, Morgan K, Gilchrist S. Does elite sport degrade sleep quality. Sports Medicine 2017; 47: 1317-1333

12. Bird SP. Sleep, recovery, and athletic performance: A brief review and recommendations. Strength Cond. J
2013; 35-43.

13. Juliff LE, Halson SL, \& Peiffer JJ. Understanding sleep disturbance in athletes prior to important competitions. J Sci Med Sport 2015; 18: 13-18.

14. Walters PH. Sleep, the athlete and performance. Strength Cond J 2002; 24: 17-24.

15. Corcoran G, Bird SP. Monitoring overtraining in athletes: A brief review and practical applications for strength and conditioning coaches. J Aust Strength Cond 2012; 20: 45-57.

16. Meeusen R, Duclos M, Foster C, Fry A, Gleeson M, Nieman $D$, Rietiens $G$. Prevention, diagnosis and treatment of the overtraining syndrome: Joint consensus statement of the European College of Sport Science and the American College of Sports Medicine. Med Sci Sports Exerc 2013; 45: 186-205.

17. Skein M, Duffield R, Edge J, Short MJ, Mundel T. Intermitten-sprint performance and muscle glycogen after $30 \mathrm{H}$ of sleep deprivation. Med Sci Sport Exerc 2011; 43 (7): 1301-11.

18. Mougin F, Simon- Rigaud ML, Davenne D, Renaud A, Garnier A. Effects of sleep disturbances on subsequent physical performance. Eur J Appl Physiol Occup Physiol 1999; 63: 77-82.

19. Reyner LA, Horne JA. Sleep restriction and serving accuracy in performance tennis players and effects of caffeine. Physiol Behav 2013; 120: 93-96.

20. Robey E, Dawson B, Halson S, Gregson W, Goodman C, Eastwood P. Sleep quantity and quality in elite youth soccer players: A pilot study. Eur J Sport SCi 2014; 14 (5): 410-417.

21. Zhao J, Tian Y, Nie J, Xu J, Liu D. Red light and the sleep quality and endurance performance of Chinese female basketball players. J Ath/ Train 2012; 47 (6): 673-8.

22. Johns MW. A new method for measuring daytime sleepiness: the Epworth sleepiness scale. Sleep 1991; 14: 540-5.

23. Buyssee DJ, Reynolds CRI, Monk TH, Berman SR, Kupfer DJ. The Pittsburgh Sleep Quality Index: A new instrument for psychiatric practice and research. Psychiatric Research 1989; 28 (2): 193-213.

24. Simpson S, Gibbs EL, Matheson GO. Reviewing optimizing sleep to maximize performance: implications and recommendations for elite athletes. Scand J Med Sci Sports 2017; 27: 266-274. 